\title{
Dental Caries Status among the Elderly in the Rural Areas of Yunnan Province, China: A Cross-Sectional Study
}

\section{Shinan Zhang}

School of Stomatology, Kunming Medical University

Hui Ding

School of Stomatology, Kunming Medical University

Xun Shen

School of Stomatology, Kunming Medical University

Lingli Pu

School of Stomatology, Kunming Medical University

Yingmei Li

School of Stomatology, Kunming Medical University

Le Cai

School of Public Health, Kunming Medical University

Lan Yao ( 940931755@qq.com)

Kunming Medical University

Research article

Keywords: dental caries, elderly, risk factors, China

Posted Date: May 12th, 2020

DOI: https://doi.org/10.21203/rs.3.rs-17204/v2

License: (c) (i) This work is licensed under a Creative Commons Attribution 4.0 International License. Read Full License 


\section{Abstract}

Background: Elder population is considered as a vulnerable group with high risk of dental caries. The older adults living in rural areas of Yunnan province account for approximately half of the province's total population. However, few studies have addressed their dental caries status. The purpose of the present study was to investigate the dental caries status and their associated factors among 65-74 years old people in the rural areas of Yunnan province, China.

Methods: This cross-sectional study recruited participants through a multistage and stratified strategy, and comprised a clinical oral examination and a questionnaire survey. Two trained and calibrated dentists, wearing LED headlights, conducted the clinical assessment with dental mirrors and CPI probes. Caries experience was measured using the DMFT and DF-Root indices. A face-to-face questionnaire survey was conducted to collect the participants' sociodemographic information, oral health-related knowledge and practices. A multi-factor ANCOVA test was used to detect the factors influencing coronal and root caries.

Results: 1175 elderly residents aged 65-74 were included. The majority of the surveyed elderly respondents (87\%) experienced coronal caries (DMFT>0), and $56 \%$ of the respondents had root caries. Their overall mean DMFT and DF-root scores were $10.4 \pm 10.9$ and $2.4 \pm 3.8$, respectively. In total, $12 \%$ of the participants were edentulous. In the final model, female $(\beta=2.872, p<0.001)$ and residents who living in less developed areas (Jianchuan district) ( $\beta=9.601$, $p<0.001)$ had higher mean DMFT scores. DF-Root scores were also higher among female $(\beta=0.739, p<0.001)$, residents who living in less developed areas (Jianchuan district) $(\beta=2.082, p<0.001)$. In addition, elderly who had lower annual family income were more likely to had more root caries $(\beta=0.642, p<$ $0.001)$.

Conclusion: The dental caries status among 65-to 74-year-old in the rural areas of Yunnan province, China, was poor. Their coronal and root caries experience both varied by gender and place of residence. Cost-effective prevention and treatment programs for caries management should be implemented to benefit the elderly population in the rural areas of Yunnan province.

\section{Background}

Although the overall oral health status has improved markedly in the world over the last few decades, the burden of dental caries among the elderly is still significant [1]. In 2016, untreated dental caries in permanent teeth remained the most common oral disease, affecting 2.4 billion people in the worldwide [2]. The prevalence of dental caries was identified to reach its third peak at around 70 years old [3]. Certainly, dental caries is a chronic cumulative disease, but it does not necessarily increase due to aging [4].

The aetiology of dental caries occurring among elderly is complex. One of the main reasons for the extensive caries status was attributed to the increased exposure of the tooth surfaces [5]. Furthermore, saliva flow and buffering capacity, qualitative and quantitative microbiological changes, and the capacity of removing dental plaque can contribute to caries occurrence in elderly [6, 7]. Due to the mineral content and organic component differences between the tooth crown and tooth root, the risk factors related to the dental caries on the crown or root are probably not exactly the same. For example, more root caries than coronal caries were found among tobacco users [8].

Carious lesions can cause pain and affect masticatory function. Without treatment, they may progress to pulp necrosis and periapical pathology, or even lead to systemic infection [9]. Severe dental caries can also lead to tooth loss, edentulism or poor nutrition, as well as impact people's self-esteem, social functioning and interaction $[10,11]$. Consequently, dental caries lead to a considerable economic burden on both individuals and society, including direct costs (e.g., diagnosis, medicine, and treatment) and indirect costs (e.g., absenteeism from work and cost for research). In 2010, the total costs for dental caries accounted for US\$442 billion globally [12].

China is the second-largest global economy, with the largest population in the world of 1.4 billion people. In 2017, China had 158 million elderly individuals aged 65 or above (11\% of the total population) [13]. According to the United Nations' aging standards, China is already considered an aging nation. The Chinese Health and Family Planning Commission predicted a massive elder population explosion in China by 2050 , with up to 400 million people aged $65+$ [14]. An upward trend in the prevalence of dental caries among the elderly population has also been observed, increasing from $65 \%$ in 1995 to $98 \%$ in 2015 , based on national oral health surveys $[15,16]$.

Currently, few studies have investigated the dental caries status among the elderly population in Yunnan province. One study conducted in 2016-2018 reported that $69 \%$ of the people aged $60-93$ years in Kunming City, the capital city of Yunnan province, experienced dental caries [17]. The participants in that study were not the index age group recommended by the World Health Organization (WHO). Therefore, comparisons with other studies were not possible. However, it is reasonable to speculate that the dental caries status among the elderly people in the rural areas of Yunnan province is even more severe. Furthermore, there is only one dentist per 20,000 people in Yunnan, which is double the ratio of China and four times the ratio recommended by the WHO [18, 19]. Thus, without proper prevention and treatment strategies, it will be a major challenge for local authorities to address dental health problems among vulnerable older adults in Yunnan, especially in rural areas.

Epidemiological studies are essential tools for stakeholders to surveil disease patterns and take immediate action for disease control and prevention. Therefore, the primary aim of the present study was to investigate the dental caries status among 65- to 74-year-olds in the rural areas of Yunnan province and to analyse the influencing factors.

\section{Methods}

This cross-sectional study, approved by the Ethics Committee of the Affiliated Stomatological Hospital of Kunming Medical University, was performed from November 2018 to May 2019. 


\section{Sample size calculation}

According to the previous study, the caries prevalence was set at $69 \%$ [17]. The sample size was 913 , with a confidence interval width of $6 \%\left(n=4 \times 1.96^{2} \times p\right.$ $\times[1-p] / L^{2}$; [n: no. of elderly, p: prevalence, and L: width of the $95 \%$ confidence interval]). We estimated a $90 \%$ response rate, and the participants needed to be at least 1014.

\section{Study design and participant selection}

Yunnan province is ethnically heterogeneous, with Han people as the predominant population. It is situated in the south-western frontiers of China, bordering Myanmar, Laos, and Vietnam. The gross domestic product of Yunnan province was ranked $20^{\text {th }}$ among the 31 provinces in China [20]. Overall, it is an undeveloped province. Based on the latest national population census, the total population in Yunnan is 46 million, with over 3 million elderly residents. Around half of the population live in rural areas [21].

The participant selection followed a multistage and stratified strategy. All rural districts in Yunnan province were invited and divided into two strata by the average per capita disposable income in Yunnan province in 2017 (US\$1400) [22]. In the first stage, two districts, Jianchuan district and Ninger district, were randomly selected to represent the two strata. In 2017, the average per capita disposable income in the rural areas of the two districts was US\$1407 and US\$1154, respectively [23]. The dentist to population ratio of Jianchuan district was 1:21,000 and 1:24,000 in Ninger district [24]. Then, all the rural villages in the selected districts were numbered. The lottery method was used to randomly select three villages from each district. In the same way, three communities from the lists of each village were randomly selected. However, The dentist to population ratio was not available for each selected village or community. All local residents aged between 65 and 74 years in these communities were invited. Participants excluded were those that were not able to comply with the clinical exam and completion of the questionnaire.

\section{Clinical examination}

Two trained and calibrated dentists, wearing headlights, carried out the clinical oral health status assessment with plane dental mirrors and ball-ended probes in a community centre in each village. Dental caries status was evaluated with the DMFT and DF-Root indices following the instructions of the WHO [25]. All permanent teeth, including the wisdom teeth, were evaluated. Coronal and root caries were recorded separately for each decayed tooth (DT). If an unmistakable cavity was present on the crown surface, it was coded as coronal caries. A filled crown with caries or a tooth with a temporary filling was recorded as coronal caries. We recorded root caries as present when a lesion felt soft or leathery on probing with the CPI probe. If any doubt existed, caries was not recorded. The categorization of missing tooth (MT) was used for teeth that were extracted due to caries or other reasons. A filled crown or filled root (FT) was recorded as tooth with filling, without caries on the crown or on the root. The intra- and inter-examiner reliability were evaluated throughout the study by re-examining approximately $10 \%$ randomly selected participants. The kappa values of the intra- and inter-examiner reliability were higher than 0.90 .

\section{Questionnaire survey}

A structured questionnaire was developed based on the previous study conducted in China [26, 27]. A pilot test was conducted before the main study. After the clinical examination, Trained interviewers assisted the participants to fill in the questionnaire during the face-to-face interviews due to the low education level of the elderly. The questionnaire consisted of three parts (Appendix 1):

(i) the elderly's socioeconomic and demographic information, specifically gender, ethnicity, education level, place of residence, marital status, and annual family income;

(ii) the elderly's oral health-related behaviours, specifically dental visit history and tooth-brushing habits; and

(iii) the elderly's oral health-related knowledge about the perceived causes and prevention of dental caries and periodontal diseases. In total, there were four multiple choice questions. For each question, all the alternatives were considered correct answers, excluding "I don't know" and "no answer". A maximum of three alternatives could be chosen for each question (one point for each correct answer). The total dental knowledge score was calculated, which ranged from 0 to 12 [27].

\section{Statistical analysis}

Before the statistical analysis, proofreading and a logic check were conducted. One research assistant conducted the data analysis using IBM SPSS Statistics version 25.0 (IBM Corp., Armonk, New York, United States). Descriptive analysis was conducted to describe the overall measurements of the dental caries, including their distribution, central tendency and dispersion. Chi-square tests were employed to compare the differences in the percentages between groups. Independent t-tests (two groups) and one-way ANOVA (more than two groups) were used to analyse the differences in dental caries experience (mean DMFT scores) between groups. When a variable with more than two subgroups was statistically associated with the caries experience (mean DMFT scores), a Bonferroni test was performed to detect the differences within the subgroups. A multifactor ANCOVA test was used to identify the factors influencing caries status. The dependent variable was the mean DMFT scores. All independent variables, such as the socioeconomic and demographic determinants and oral health-related knowledge scores, were entered into the model. An alpha of 0.05 was used as the cut-off for statistical significance.

\section{Results}

This study was conducted at the community centres of the two selected districts. The ratio of participants from both districts was around 1:1. Initially, a total of 1205 residents were recruited. Of them, 10 elderly could not cooperate and 20 participants were absent from the study. The final study sample comprised 
1175 persons. The response rate for the study was $98 \%$. There were $524(44 \%)$ male and $651(66 \%)$ female participants. A majority of them were married or cohabiting (76\%). Approximately half of them (53\%) had received primary education. Around two- thirds of them (70\%) belonged to ethnic minorities.

As illustrated in Table 1, a large number of the participants (87\%) experienced dental caries (DMFT>0), and $55 \%$ of them had root caries. Their overall mean DMFT and DF-Root scores ( \pm SD) were $10.4 \pm 10.9$ and $2.4 \pm 3.8$, respectively. More than half of the carious teeth were untreated or had secondary caries (68\%). Only $12 \%$ of the participants had fillings without caries, and their mean FT score was $0.3 \pm 1.2$. Among the participants, almost half of them (47\%) had missing teeth. In total, $12 \%$ of the participants were edentulous.

A majority of the participants (91\%) had a daily tooth-brushing habit. Almost half of them (45\%) brushed their teeth for around one minute. A large number of the respondents (77\%) had no experience with dental attendance. Table 2 illustrates the association between the mean DMFT /DF-Root scores and the studied variables. Higher mean DMFT/DF-Root scores were found in women than men $(p<0.001)$. Ethnic minority people experienced more dental caries than the Han people $(p<0.001)$. Participants living in Jianchuan district had higher mean DMFT/DF-Root scores than inhabitants of the other district $(p<0.001)$. Education level was not related to the caries experience $(p=0.074)$. The highest mean DMFT/DF-Root scores were also found in the elderly with annual family income between RMB 15,000 and 30,000 ( $<<0.001)$. Participants who did not brush their teeth daily had higher mean DMFT scores $(p=0.003)$. Dental attendance experience was not related to the coronal or root caries experience $(p>0.05)$. Respondents with some dental knowledge experience had lower mean DMFT scores than those who had none $(p<0.001)$. However, the mean DF-Root scores were not affected by the oral health-related knowledge status $(p=0.057)$.

In the final multivariate model using ANCOVA analysis (Table 3), higher mean DMFT scores were detected among elderly women ( $\beta=2.872, p<0.001)$ and participants who living in less developed areas (Jianchuan district) $(\beta=9.601, p<0.001)$. Mean DF-Root scores were higher among females $(\beta=0.739, p<$ $0.001)$, residents who living in Jianchuan district $(\beta=2.082, p<0.001)$, and those had lower annual family income (less than $15,000 \mathrm{RMB})(\beta=0.642, \mathrm{p}<$ $0.001)$.

\section{Discussion}

As life expectancy rises, the older adults are a rapidly growing proportion of the Yunnan population [28]. With aging, exposure to risk factors may increase the progression of dental caries. In the present study, coronal and root caries among the elderly in the rural areas of Yunnan province were prevalent. The majority of the participants had poor oral health knowledge. Few of them had visited a dentist in their lifetime, and most of the caries were untreated. Gender and place of residence were the only risk factors for coronal or root caries.

In this study, the majority of the participants had experienced dental caries (87\%), although this percentage was lower than that reported in the national survey (98\%) [16]. However, it was significantly higher than the percentage in Sichuan (77\%) and the north-eastern provinces of China (64\%) [29, 30]. Previous studies showed differences in the prevalence of root caries among the elderly worldwide, ranging from $25 \%$ to $100 \%$ [31]. The prevalence of root caries in our study $(55 \%)$ was found to be lower than that reported in the national survey $(64 \%)$. However, the dental caries burden in both crowns and roots was still heavy among the elderly in the rural areas of Yunnan province.

It should be noted that the prevalence of the edentulouness in this study $(12 \%)$ was higher than the prevalence reported in China national oral health survey (4.5\%) [32] and in the United States'National Health Survey (4.9\%) [33]. Tooth loss is a robust measurement of the combined burden of untreated dental caries or unsuccessfully treated periodontal disease [34]. Our results show that this high prevalence of edentulism could mainly be caused by the lack of prevention and treatment for both dental caries and periodontal disease. As mentioned before, the financial barriers and limited access to oral health care could have contributed to their poor oral health status. Therefore, oral health strategies should be implemented to provide accessible and affordable dental care services throughout rural areas to further improve tooth retention.

Although many previous studies have reported a negative correlation between education level and caries, a statistically significant correlation was not detected in this study [35]. This may be due to the generally low education level among the participants. Meanwhile, we found that the most of the participants had inadequate dental knowledge, even though the national oral health education programs, such as the "Love Teeth Day" campaign [36], were carried out every year to pass on the oral health care knowledge. This study showed that changes should be considered when providing oral health promotion programs, focusing on oral health knowledge and awareness, as well as the importance of regular visits to the dentist, especially for those with a low education level. Considering the shortage of dentists in Yunnan, oral health policymaker should ensure the challenge in providing professional dental care. Education courses should be initiated to train dental hygienists and therapists in Yunnan to supplement local oral health care team.

In the final model, gender was significantly associated with the caries experience. Women were more likely to experience dental caries on either crown or root than men. This was consistent with many previous studies $[16,30]$. One explanation is the earlier tooth eruption among women than men. This prolonged tooth exposure in the oral environment could increased susceptibility to dental caries. The traditional belief in China that women should not brush their teeth in the first month after childbirth could also be a factor that leads to their higher caries rate.

Ethnicity is usually considered a risk factor for dental caries [37]. In this study, the influence of ethnicity on dental caries experience was less than that of geographic variable. Therefore, it was not included in the final model. People living in the less developed district (Jianchuan district) had higher mean DMFT and DF-Root scores than the participants living in Ninger district. Considerable inequalities in dental caries due to the place of residence were also observed among the older population in other parts of China [16].

In this study, we reported the caries status using both the DMFT and DF-Root indices, which was consistent with the latest national oral health survey and most previous studies $[4,16]$. Thus, the results could be easily compared. However, in many cases, we were unsure if teeth were missing due to caries or 
periodontal diseases. In addition, several root restorations had been performed, probably due to the previous presence of a non-carious cervical lesion (abrasion, abrasion, or erosion), so dental caries experiences could be overestimated.

This study had a high response rate, due to the support of the local health department. However, only two districts from the rural areas in Yunnan were included. The homogeneity within districts and heterogeneity between districts may affect the representativeness of the estimate. Furthermore, massive internal urbanization occurred in the 1980s [38]. Elderly individuals with immigration experiences could access better oral health services in urban cities. This might have had a positive effect on their oral health status. However, this confounding factor was not investigated in the present study. Meanwhile, according to the available data in Yunnan, 2\% of the elderly were living in long-term care facilities but they were not included in this study [13]. We also excluded the elderly who were not able to complete the study. Therefore, the results of this study should still be interpreted with caution.

\section{Conclusion}

The dental caries status among 65-to 74-year-old in the rural areas of Yunnan province, China, was poor. Their coronal and root caries experience both varied by gender and place of residence. In addition, low economic status was a risk factor for root caries. Cost-effective prevention and treatment programs for caries management should be implemented to benefit the elderly population in the rural areas of Yunnan province.

\section{List Of Abbreviations}

WHO (World Health Organization)

\section{Declarations}

\section{Ethics approval and consent to participate}

This study was approved by the Ethics Committee of the Affiliated Stomatological Hospital of Kunming Medical University. Written consent forms were received from the participants before the fieldwork.

\section{Consent for publication}

Not applicable

\section{Availability of data and materials}

The data set generated and/or analyzed during the current study are available from the first author on reasonable request.

\section{Competing interests}

The authors declare that they have no competing interests.

\section{Funding}

This study was supported by the Yunnan Provincial Science and Technology Department and Kunming Medical University Conjoint Fund \#2018FE001(-073).

\section{Acknowledgment}

The authors appreciated the participation of the elderly in this study. We also would like to express our gratitude to the Yunnan Centre for Disease Control and Prevention, and the local government of Jianchuan and Ninger for their support. We also appreciated all the research assistants who contributed to this study.

\section{Author information}

Affiliations

Department of Dental Public Health, School of Stomatology, Kunming Medical University, Kunming City, Yunnan Province, China

Shinan Zhang

Department of Pediatric Dentistry, School of Stomatology, Kunming Medical University, Kunming City, Yunnan Province, China

Hui Ding

Department of Prosthetic Dentistry, School of Stomatology, Kunming Medical University, Kunming City, Yunnan Province, China

Xun Shen

School of Stomatology, Kunming Medical University, Kunming City, Yunnan Province, China

Lingli Pu

School of Stomatology, Kunming Medical University, Kunming City, Yunnan Province, China 
Yingmei Li

School of Public Health, Kunming Medical University, Kunming City, Yunnan Province, China

Le Cai

School of Stomatology, Kunming Medical University, Kunming City, Yunnan Province, China

Lan Yao

Contribution

SZ and HD conducted the data analysis and prepared the manuscript. XS and LC designed and cooperated in the fieldwork. LP and YL assisted in data collection. LY performed critical revision of the manuscript. All the authors read, revised and approved the final manuscript.

\section{References}

1. Kassebaum NJ, Smith AGC, Bernabé E, Fleming TD, Reynolds AE, Vos T, et al. Global, regional, and national prevalence, incidence, and disability-adjusted life years for oral conditions for 195 Countries, 1990-2015: a systematic analysis for the global burden of diseases, injuries, and risk factors. J Dent Res. 2017;96(4):380-7.

2. Kassebaum NJ, Bernabé E, Dahiya M, Bhandari B, Murray CJL, Marcenes W. Global burden of untreated caries: a systematic review and metaregression. J Dent Res. 2015;94(5):650-8.

3. Lamster IB, Asadourian L, Del Carmen T, Friedman PK. The aging mouth: differentiating normal aging from disease. Periodontol 2000. $2016 ; 72(1): 96-107$.

4. Tonetti MS, Bottenberg P, Conrads G, Eickholz P, Heasman P, Huysmans M-C, et al. Dental caries and periodontal diseases in the ageing population: call to action to protect and enhance oral health and well-being as an essential component of healthy ageing - consensus report of group 4 of the joint EFP/ORCA workshop on the boundaries between caries and periodontal diseases. J Clin Periodontol. 2017;44(S18):S135-S44.

5. López R, Smith PC, Göstemeyer G, Schwendicke F. Ageing, dental caries and periodontal diseases. J Clin Periodontol. 2017;44(S18):S145-S52.

6. Chapple ILC, Bouchard P, Cagetti MG, Campus G, Carra M-C, Cocco F, et al. Interaction of lifestyle, behaviour or systemic diseases with dental caries and periodontal diseases: consensus report of group 2 of the joint EFP/ORCA workshop on the boundaries between caries and periodontal diseases. $J$ Clin Periodontol. 2017;44(S18):S39-S51.

7. Yildiz G, Ermis RB, Calapoglu NS, Celik EU, Türel GY. Gene-environment interactions in the etiology of dental caries. J Dent Res. 2016;95(1):74-9.

8. Zhang J, Sardana D, Wong M, Leung KCM, Lo E. Factors associated with dental root caries: a systematic review. JDR Clinical \& Translational Research. 2019;5:238008441984904.

9. Peres M, Macpherson L, Weyant R, Daly B, Venturelli R, Mathur M, et al. Oral diseases: a global public health challenge. The Lancet. 2019; $394: 249-60$.

10. Saito M, Shimazaki Y, Nonoyama T, Tadokoro Y. Type of dental visit and number of remaining teeth in Japanese elders. J Oral Sci. 2018; 60: 611-7.

11. Wu LL, Cheung KY, Lam PYP, Gao X. Oral health indicators for risk of malnutrition in elders. J Nutr Health Aging. 2018;22(2):254-61.

12. Listl S, Galloway J, Mossey P, Marcenes W. Global economic impact of dental diseases. J Dent Res. 2015; 94(10):1355-61.

13. National Bureau of Statistics of the People's Republic of China. 2019 China statistical yearbook (in Chinese). Available at: http://www.stats.gov.cn/tjsj/ndsj/2019/indexch.htm. Accessed: 26 February, 2020.

14. Fang EF, Scheibye-Knudsen M, Jahn H, Li J, Ling L, Guo H, et al. A research agenda for aging in China in the 21st century. Ageing Res Rev. 2015;24(Pt B):197-205.

15. Wang HY, Petersen PE, Bian JY, Zhang BX. The second national survey of oral health status of children and adults in China. Int Dent J. 2002; 52:283-90.

16. Gao Y, Hu T, Zhou X, Shao R, Cheng R, Wang G, et al. Dental caries in Chinese elderly people: findings from the 4th national oral health survey. Chin J Dent Res. 2018; 21:213-20.

17. Xie LY. The study on tooth defect/missing and restoration and its influential factors among elderly people from Kunming. Kunming: Kunming Medical University, 2018. https://kns.cnki.net/KCMS/detail/detail.aspx?

dbcode=CMFD\&dbname=CMFD201901\&filename=1018288360.nh\&uid=WEEvREcwSIJHSIdRa1FhcTdnTnhXS1B5MmpjalU2Nkh1WFI4YkVyb0NBST0=\$9 Accessed 26 February, 2020.

18. Gao BD, LI G, Wang Y, Guo J, Hao YY, Ding XY. The investigation on dentist structure in some provinces and a city of China (in Chinese). J Pract Stomatol. 2011, 27(2):279-282.

19. Ministry of Health of the People's Republic of China. 2009 China health statistical yearbook (in Chinese). 1st ed. Beijing: Peking Union Medical College Press, 2010.

20. Yao DQ. Competition of GDP growth rate among the 31 provinces: the richest and the last. China economic weekly. 2019(04):30-3.

21. National Bureau of Statistics of the People's Republic of China. 2013 China statistical yearbook (in Chinese). 1st ed. Beijing: Statistical Publishing House; 2013.

22. Statistical Bureau of Yunnan Province. 2018 Yunnan survey yearbook (in Chinese). 1st ed. Beijing: Statistical Publishing House; 2018.

23. Statistical Bureau of Yunnan Province. 2018 Yunnan survey yearbook (in Chinese). 1st ed. Beijing: Statistical Publishing House; 2018. 
24. Zhang W. Research on the status of stomatological medical institutions and human resources in Yunnan Province. Kunming: Kunming Medical University, 2012.https://kns.cnki.net/KCMS/detail/detail.aspx?

dbcode=CMFD\&dbname=CMFD2012\&filename=1012410776.nh\&uid=WEEvREcwSIJHSIdTTEYzVnBFak5MTmt6czZrK1pqeXo4NWtTb1IXSmJ6cz0=\$9A4 Accessed 19 April, 2020.

25. World Health Organization (WHO). Oral health surveys: basic methods, 5th edition. Geneva: World Health Organization; 2013. Available at: http://www.who.int/oral_health/publications/9789241548649/en/. Accessed 26 February, 2020.

26. Zhang S, Xu B, Liu J, Lo E, Chu C-H. Dental and periodontal status of 12-year-old Dai school children in Yunnan Province, China: a cross-sectional study. BMC Oral Health. 2015;15

27. Lin HC, Wong MCM, Wang ZJ, Lo ECM. Oral health knowledge, attitudes, and practices of Chinese adults. J Dent Res. 2001;80(5):1466-70.

28. Partridge L, Deelen J, Slagboom PE. Facing up to the global challenges of ageing. Nature. 2018;561(7721):45-56.

29. Yin W, Yang Y-M, Chen H, Li X, Wang Z, Cheng L, et al. Oral health status in Sichuan Province: findings from the oral health survey of Sichuan, $2015-2016$. Int J Oral Sci. 2017;9(1):10-5.

30. Liu L, Zhang Y, Wu W, Cheng M, Li Y, Cheng R. Prevalence and correlates of dental caries in an elderly population in northeast China. PLoS One. 2013;8(11): e78723-e.

31. Gavriilidou N, Belibasakis G. Root caries: the intersection between periodontal disease and dental caries in the course of ageing. Br Dent J. 2019; 227:1063-7.

32. Guo J, Ban JH, Li G, Wang X, Feng XP, Tai BJ et al. Status of tooth loss and denture restoration in Chinese adult population: findings from the 4th national oral health Survey. Chin J Dent Res. 2018;21(4):249-57.

33. Slade GD, Akinkugbe A, Sanders A. Projections of U.S. edentulism prevalence following 5 decades of decline. J Dent Res. $2014 ; 93$.

34. Department of Health. Oral health survey 2011. 1st ed. Hong Kong: Government Logistics Department; 2013.

35. Wang L, Cheng L, Yuan B, Hong X, Hu T. Association between socio-economic status and dental caries in elderly people in Sichuan Province, China: a cross-sectional study. BMJ Open. 2017;7(9): e016557.

36. Lin HC, Schwarz E. Oral health and dental care in modern-day China. Community Dent Oral Epidemiol. 2001;29(5):319-28.

37. Zhang S, Li Y, Liu J, Wang W, Ito L, Li SKY, et al. Dental caries status of Lisu preschool children in Yunnan Province, China: a cross-sectional study. BMC Oral Health. 2019; 19:17.

38. Yan LY. Filial peity in flow-A comparative study of three mobile generetions from a family in central Henan Province. Shanghai: East China University of Science and Technology, 2019. https://kns.cnki.net/KCMS/detail/detail.aspx? dbcode=CMFD\&dbname=CMFD202001\&filename=1019018965.nh\&v=MDMwMjZZUzdEaDFUM3FUcldNMUZyQ1VSN3FmWmVSb0Z5cmhWN3pJVkYyNk Accessed 26 February, 2020.

\section{Tables}

Table 1 Dental caries status of the elderly

\begin{tabular}{lcc}
\hline Dental caries & Prevalence Mean (SD) \\
\hline Caries experience (DMFT>0) & $87 \%$ & $10.4(10.9)$ \\
Decay teeth (DT) & $68 \%$ & $3.2(4.1)$ \\
Missing teeth (MT) & $47 \%$ & $6.9(10.8)$ \\
Filled teeth (FT) & $12 \%$ & $0.3(1.2)$ \\
DF-Root & $55 \%$ & $2.4(3.8)$ \\
\hline
\end{tabular}

Table 2 Dental caries experience (DMFT/DF-Root) and studied variables 


\begin{tabular}{|c|c|c|c|c|}
\hline$\overline{\text { Variables }(\%, \mathbf{N})}$ & DMFT (SD) & p-value & DF-Root (SD) & p-value \\
\hline$\overline{\text { Gender }}$ & & $<0.001$ & & 0.003 \\
\hline Male $(44 \%, 524)$ & $9.1(10.2)$ & & $2.1(3.3)$ & \\
\hline Female $(66 \%, 651)$ & $11.5(11.3)$ & & $2.7(4.0)$ & \\
\hline Ethnicity & & $<0.001$ & & $<0.001$ \\
\hline Han $(30 \%, 351)$ & $6.6(10.2)$ & & $1.3(2.4)$ & \\
\hline Ethnic minority $(70 \%, 824)$ & $12.0(10.7)$ & & $2.9(4.1)$ & \\
\hline Place of residence & & $<0.001$ & & $<0.001$ \\
\hline Jianchuan District $(45 \%, 533)$ & $15.6(10.0)$ & & $3.5(4.5)$ & \\
\hline Ninger District $(55 \%, 642)$ & $6.1(9.7)$ & & $1.5(2.7)$ & \\
\hline Marital status & & 0.974 & & 0.454 \\
\hline Married or cohabitation $(76 \%, 893)$ & $10.4(10.6)$ & & $2.5(3.8)$ & \\
\hline Single, divorced or Widowed $(24 \%, 282)$ & $10.4(11.6)$ & & $2.3(3.7)$ & \\
\hline Education level & & 0.074 & & 0.295 \\
\hline Illiterate $(28 \%, 330)$ & $11.5(11.3)$ & & $2.6(4.0)$ & \\
\hline Primary $(53 \%, 619)$ & $9.8(10.7)$ & & $2.4(3.6)$ & \\
\hline Secondary or above $(19 \%, 226)$ & $10.3(10.5)$ & & $2.1(3.8)$ & \\
\hline Annual family income (RMB) & & $0.001(\mathrm{a}=\mathrm{c}<\mathrm{b})$ & & $0.033(\mathrm{a}=\mathrm{c}<\mathrm{b})$ \\
\hline$<15,000^{\mathrm{a}}(31 \%, 368)$ & $9.5(10.7)$ & & $2.4(3.4)$ & \\
\hline $15,000-30,000^{\mathrm{b}}(37 \%, 435)$ & $11.9(11.2)$ & & $2.7(4.1)$ & \\
\hline$>30,000^{\mathrm{C}}(32 \%, 372)$ & $9.5(10.4)$ & & $2.1(3.7)$ & \\
\hline Daily toothbrushing & & 0.003 & & 0.609 \\
\hline No $(9 \%, 107)$ & $13.4(12.5)$ & & $2.6(4.4)$ & \\
\hline Yes $(91 \%, 1068)$ & $10.1(10.6)$ & & $2.4(3.7)$ & \\
\hline Tooth brushing time & & 0.236 & & 0.051 \\
\hline$\leq 1 \min (45 \%, 488)$ & $9.7(10.5)$ & & $2.5(3.8)$ & \\
\hline $2-3 \min (50 \%, 544)$ & $9.9(10.8)$ & & $2.1(3.4)$ & \\
\hline$>3 \min (5 \%, 58)$ & $12.2(10.0)$ & & $3.0(3.7)$ & \\
\hline Dental attendance experience & & 0.608 & & 0.163 \\
\hline Never $(77 \%, 904)$ & $10.5(11.1)$ & & $2.5(3.9)$ & \\
\hline More than once $(23 \%, 271)$ & $10.1(9.9)$ & & $2.1(3.3)$ & \\
\hline Oral health-related knowledge & & $<0.001$ & & 0.057 \\
\hline Score $=0(60 \%, 704)$ & $12.0(11.2)$ & & $2.6(3.9)$ & \\
\hline Score $>0(40 \%, 471)$ & $8.1(10.0)$ & & $1.8(2.3)$ & \\
\hline
\end{tabular}

Table 3 Relationship between dental caries experience and selected independent variables (final model of multi-factor ANCOVA)

${ }^{\mathrm{a}}$ Reference Category

Adjusted $\mathrm{R}^{2}=0.204$ (coronal caries) and 0.083 (root caries)

\section{Figures}




\begin{tabular}{|c|c|c|c|c|c|}
\hline & Independent variables & Group & Beta SE & Eta-square & $\overline{\text { p-value }}$ \\
\hline \multirow{5}{*}{$\begin{array}{l}\text { Coronal caries } \\
\text { (Mean DMFT scores) }\end{array}$} & Gender & Female & 2.8720 .569 & 0.210 & $<0.001$ \\
\hline & & $\mathrm{Male}^{\mathrm{a}}$ & & & \\
\hline & Place of residence & Jianchuan District & 9.6010 .569 & 0.196 & $<0.001$ \\
\hline & & Ninger District ${ }^{\mathrm{a}}$ & & & \\
\hline & Intercept & & 4.4540 .504 & & $<0.001$ \\
\hline \multirow{8}{*}{$\begin{array}{l}\text { Root caries } \\
\text { (Mean DF-Root scores) }\end{array}$} & Gender & Female & 0.7390 .211 & 0.010 & $<0.001$ \\
\hline & & Male $^{\mathrm{a}}$ & & & \\
\hline & Place of residence & Jianchuan District & 2.0820 .215 & 0.074 & $<0.001$ \\
\hline & & Ninger District $^{\mathrm{a}}$ & & & \\
\hline & Annual family income (RMB) & $<15000$ & 0.6420 .267 & 0.005 & 0.034 \\
\hline & & $15000-30000$ & 0.5250 .254 & 0.004 & \\
\hline & & $>30000^{\mathrm{a}}$ & & & \\
\hline & Intercept & & 0.6620 .244 & & $<0.001$ \\
\hline
\end{tabular}

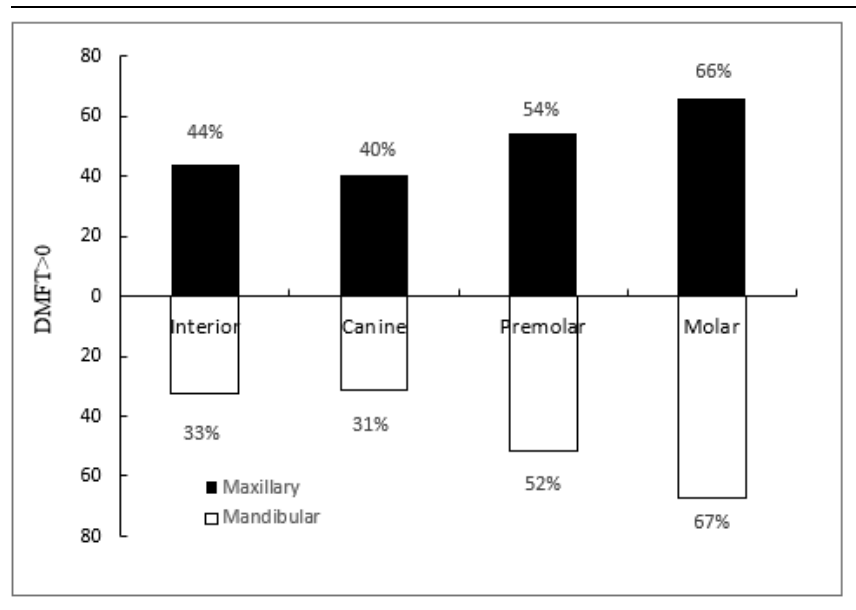

Figure 1

Prevalence of dental caries (DMFT $>0$ ) by teeth type

\section{Supplementary Files}

This is a list of supplementary files associated with this preprint. Click to download.

- questionnaire.docx 